\title{
Downregulation of NPM reverses multidrug resistance in human hepatoma cells via inhibition of P-glycoprotein expression
}

\author{
FEI LUO ${ }^{1 *}$, HUIYU LI ${ }^{2 *}$, JIANFANG LIANG $^{3}$, HONGYAN JIA ${ }^{4}$, XIAOYU LI $^{5}$, \\ HONG XIAO $^{3}$, XUEHUA HE ${ }^{6}$, JIEFENG HE ${ }^{2}$, YANZHANG TIAN ${ }^{2}$ and HAOLIANG ZHAO ${ }^{2}$ \\ ${ }^{1}$ Department of Breast Surgery, Shanxi Cancer Hospital, Taiyuan, Shanxi 030013; ${ }^{2}$ Department of General Surgery, \\ Shanxi Academy of Medical Sciences, Shanxi Dayi Hospital, Taiyuan, Shanxi 030032; Departments of \\ ${ }^{3}$ Pathology and ${ }^{4}$ General Surgery, The First Hospital of Shanxi Medical University, Taiyuan, Shanxi 030001; \\ ${ }^{5}$ Department of Molecular Biology, Shanxi Cancer Hospital, Taiyuan, Shanxi 030013; ${ }^{6}$ Department of Blood Transfusion, \\ Shanxi Academy of Medical Sciences Shanxi Dayi Hospital, Taiyuan, Shanxi 030032, P.R. China
}

Received December 11, 2015; Accepted December 19, 2016

DOI: $10.3892 / \mathrm{mmr} .2017 .6246$

\begin{abstract}
Multidrug resistance (MDR) is an important issue in current cancer treatments. In human cancer, drug resistance is primarily associated with the overexpression of multidrug resistance gene 1 (MDR1). Therefore, the human MDR1 gene promoter may be a target for anti-MDR drug screening. Numerous methods to prevent MDR have been investigated. However, they have been proven to be clinically ineffective. Therefore, the aim of the present study was to investigate whether downregulation of nucleophosmin (NPM) demonstrates any effects on the reversal of MDR in hepatocellular carcinoma (HCC) cells. In the present study, two in vitro MDR HCC cell lines, HepG2/Adriamycin (ADM) and SMMC7721/ ADM, were established and the level of MDR was measured. The results demonstrated that NPM downregulation markedly reversed the effects of MDR in the model used. In addition, NPM downregulation reduced P-glycoprotein expression, as well as MDR1 expression. These results suggested that downregulation of NPM may be a novel and effective method of reversing the effects of MDR, and may be a potential adjuvant for tumor chemotherapy.
\end{abstract}

Correspondence to: Dr Haoliang Zhao, Department of General Surgery, Shanxi Academy of Medical Sciences, Shanxi Dayi Hospital, 99 Longcheng Main Street, Taiyuan, Shanxi 030032, P.R. China

E-mail: haoliangzhao@hotmail.com

*Contributed equally

Abbreviations: MDR, multidrug resistance; MDR1, multidrug resistance gene 1; P-gp, P-glycoprotein; HCC, hepatocellular carcinoma; NPM, nucleophosmin

Key words: hepatocellular carcinoma, multidrug resistance, nucleophosmin, p-glycoprotein, NSC348884

\section{Introduction}

Hepatocellular carcinoma (HCC) is one of the most common types of cancer worldwide (1), and is currently the third leading cause of cancer-associated death (2). The majority of patients with HCC are not suitable candidates for surgery as they are diagnosed at an advanced stage. Chemotherapy with cytotoxic drugs, including anthracyclines, fluoropyrimidines and platinum complexes, serve a significant role in the management of terminal HCC. However, patients with HCC often do not respond to chemotherapy due to the development of multidrug resistance (MDR). Therefore, research into the development of a safe and effective MDR reversal agent is urgently required.

Nucleophosmin (NPM) is a major nucleolar phosphoprotein that has been implicated in multiple cellular functions, including ribosomal protein assembly and transport $(3,4)$, centrosome duplication (5-7), molecular chaperone activity to prevent protein aggregation $(8,9)$ and regulating the activity of the tumor suppressors $\mathrm{p} 53$ (10-12) and $\mathrm{p} 14^{\mathrm{ARF}}(13-15)$. Previous studies have demonstrated that the level of NPM expression is markedly increased when cells are committed to mitogenesis $(16,17)$. In addition, excessive NPM expression has been linked to cellular transformation and oncogenesis (18). NPM overexpression is often observed in human cancers, including those of the stomach (19), colon (20), bladder (21), prostate (22), thyroid (23), ovary (24), myeloid and lymphoid cells (25). It has been demonstrated that NPM overexpression in bladder cancer is independently associated with recurrence and progression to more advanced stages, which suggests that overexpression of NPM may be an important prognostic indicator for cancer recurrence (21). These findings suggest that NPM may be involved in the regulation of cellular growth in normal and neoplastic cells. Thus, it may have potential as a clinical indicator in cancer patients (21). However, it remains unknown whether NPM may regulate cellular growth in MDR HCC cell lines.

One of the most important and extensively studied mechanisms of MDR in cancer cells is the efflux mechanism, which is based on P-glycoprotein (P-gp) function $(26,27)$. P-gp is a $170 \mathrm{kDa}$ plasma membrane glycoprotein encoded by the 
human multidrug resistance gene 1 (MDR1) gene, which functions as an adenosine triphosphate (ATP)-binding cassette transporter (26). P-gp is a drug efflux pump that removes a number of chemotherapeutic drugs from MDR cancer cells (27). In addition to producing drug resistance at a cellular level, P-gp has also been demonstrated to alter the pharmacokinetics of numerous drugs and has been correlated with poor bioavailability (28-30). Therefore, P-gp inhibition may lead to the reversal of MDR during treatment with chemotherapeutic agents, and may lead to successful chemotherapy results in patients with MDR tumors (31). However, the association between P-gp and NPM in MDR HCC is currently unknown.

In the present study, the authors hypothesized that downregulated expression of NPM may increase the uptake and retention of chemotherapeutic agents via the inhibition of MDR1 expression and altered expression of P-gp in MDR HCC cells. Therefore, the aim of the present study was to investigate the cellular mechanisms of NPM-mediated reversal of MDR in HCC cells, which may re-sensitize the MDR HCC cells to chemotherapy. This novel strategy used the downregulated expression of NPM as a targeted tool in combination with chemotherapeutic agents for optimal therapeutic efficacy.

\section{Materials and methods}

Cell culture. The human HCC cell lines, HepG2 and SMMC7721, were purchased from the Institute of Biochemistry and Cell Biology (Shanghai Institutes for Biological Science, Chinese Academy of Sciences, Shanghai, China). HepG2 was cultured in Dulbecco's modified Eagle's medium (DMEM; Hyclone; GE Healthcare Life Sciences, Logan, UT, USA) and SMMC7721 was cultured in RPMI-1640 (Hyclone; GE Healthcare Life Sciences). The media were supplemented with $10 \%$ fetal bovine serum (FBS; Hyclone; GE Healthcare Life Sciences).

Multidrug resistance human HCC cell lines, HepG2/ADM and SMMC7721/ADM, were developed by the Department of General Surgery, Shanxi Dayi Hospital, Taiyuan, China). HepG2 and SMMC7721 cells were plated in a 6-well plate at a concentration of $2 \times 10^{6}$ in $2 \mathrm{ml}$ of medium. To develop the HepG2/ADM and SMMC7721/ADM cells lines, ADM (Shanghai Pharmaceuticals Holding Co. Ltd, Shanghai, China) was added respectively to HepG2 and SMMC7721 cells at increasing concentrations from 0.01 to $0.2 \mathrm{mg} / 1$ over 10 months. MDR was maintained by culturing the cells in the presence of $0.2 \mathrm{mg} / \mathrm{l} \mathrm{ADM}$. MDR HCC cells were termed HepG2/ADM and SMMC7721/ADM.

Cell viability assay. HepG2, HepG2/ADM, SMMC7721 and SMMC7721/ADM cells were plated into 96-well plates at the density of $1 \times 10^{4}$ cells $/ \mathrm{ml}$ medium. When the cells were $80 \%$ confluent, they were cultured in the presence of ADM, diamminedichloroplatinum (DDP), fluorouracil (5-Fu), vincristine sulfate (VCR) or etoposide (VP-16) for $48 \mathrm{~h}$ at $37^{\circ} \mathrm{C}$ in an incubator containing $5 \% \mathrm{CO}_{2}$. The cells were respectively treated with $0,0.1,1,10,20,30 \mathrm{mg} / 1$ ADM, DDP, VCR and 0 , $1,10,20,30,40 \mathrm{mg} / 15-\mathrm{Fu}$ and VP-16 in the presence of $10 \%$ serum medium. DDP, 5-Fu, VCR and VP-16 were purchased from Sigma-Aldrich (Merck KGaA, Darmstadt, Germany). In addition, cells were cultured in the presence of the NPM inhibitor, NSC348884 (Sigma-Aldrich; Merck Millipore, Darmstadt, Germany). Cell lines HepG2/ADM+NSC348884 and SMMC7721/ADM+NSC348884 were cultured in DMEM or RPMI-1640 containing 10\% FBS and $0.2 \mathrm{mg} / 1 \mathrm{ADM}$, together with $1,2,3,4,5$ or $6 \mu \mathrm{mol} / 1 \mathrm{NSC} 348884$. Cell proliferation was determined using a cell counting kit- 8 assay (CCK-8; Dojindo Molecular Technologies, Inc., Kumamoto, Japan). A total of $100 \mathrm{ul}$ cell suspension was added into one well of a 96-well culture plate, and 10 ul CCK- 8 was then added into the well to measure cell proliferation following medication, and absorbance was measured at a wavelength of $540 \mathrm{~nm}$ on a plate reader (PerkinElmer Wallac 1420 Victor2, Waltham, MA, USA). Data were expressed as the percentage of the survival of control, calculated from the absorbance and corrected for background. The half maximal inhibitory concentration $\left(\mathrm{IC}_{50}\right)$ was estimated by the dose of drug that resulted in $50 \%$ decrease in cell viability.

Flow cytometric analysis of cell cycle distribution. Cultured HepG2/ADM and SMMC7721/ADM cells and their parental cells were collected via trypsinization, washed with ice-cold PBS, centrifuged at $500 \times \mathrm{g}$ for $5 \mathrm{~min}$ at $4^{\circ} \mathrm{C}$, washed twice with ice-cold PBS and fixed in $70 \%$ ethanol for $2 \mathrm{~h}$ at $4^{\circ} \mathrm{C}$. Samples were rehydrated with PBS and the cells were incubated for $30 \mathrm{~min}$ at room temperature with a propidium iodide staining solution in PBS containing $0.2 \mathrm{mg} / \mathrm{ml}$ propidium iodide, $0.2 \mathrm{mg} / \mathrm{ml}$ DNAse-free RNAse A (Roche Diagnostics, Basel, Switzerland), and $0.1 \%$ Triton X-100. Using red propidium DNA fluorescence, 20,000 events were acquired with an Epics ${ }^{@}$ XL Beckman Coulter FACS machine (Beckman Coulter Inc., Brea, CA, USA) for each sample and the percentage of cells in G0/G1, S and G2/M phases of the cell cycle was calculated using the System II $^{\mathrm{TM}}$ software (Beckman Coulter Inc.) (32).

Western blot analysis. The cells were lysed at $4{ }^{\circ} \mathrm{C}$ in a lysis buffer (Cell Signaling Technology Inc., Danvers, MA, USA). The cell lysates were centrifuged at $21,000 \times \mathrm{g}$ for $15 \mathrm{~min}$ at $4^{\circ} \mathrm{C}$. The protein concentration in the supernatant was detected using a BCA kit. Then proteins from tissue homogenate were loaded on sodium dodecyl sulfate-polyacrylamide gel (12\% SDS-PAGE), transferred onto a polyvinylidene membrane, blocked with bovine serum albumin, and then incubated using the primary antibodies anti-NPM (catalog no. 3542; 1:1,000; Cell Signaling Technology Inc.), anti-MDR-1 (catalog no. 13342; 1:1,000; Cell Signaling Technology Inc.), anti-P-gp (catalog no. A10436R; 1:500; Beijing Solarbio Science \& Technology Co., Ltd, Beijing, China) and anti- $\beta$-actin (catalog no. A10938R; 1:1,000; Beijing Solarbio Science \& Technology Co., Ltd) at $4^{\circ} \mathrm{C}$, overnight. Membranes were washed three times and then incubated with horseradish peroxide -conjugated secondary antibody (catalog no. 7074S; 1:2,000; Cell Signaling Technology Inc.) for $40 \mathrm{~min}$ at room temperature. Specific antibody binding was detected using electrochemiluminescence (Chemi Doc XRS+ Imaging system, Bio-Rad Laboratories, Inc. Hercules, CA, USA). The abundance of western blot signaling was determined using the image analysis software (Chemi Doc XRS+ Imaging system, Bio-Rad Laboratories, Inc.). Western blot analysis was carried out as described previously (33). 
Table I. Determination of the $\mathrm{IC}_{50}$ values of different anticancer drugs in multidrug-resistant hepatocellular carcinoma cells.

\begin{tabular}{lcccccc}
\hline $\begin{array}{l}\text { Anticancer } \\
\text { drug }\end{array}$ & $\begin{array}{c}\text { HepG2 } \\
(\mathrm{mg} / \mathrm{l})\end{array}$ & $\begin{array}{c}\text { HepG2/ADM } \\
(\mathrm{mg} / \mathrm{l})\end{array}$ & $\begin{array}{c}\text { HepG2/ADM+ } \\
\text { NSC348884 }(\mathrm{mg} / \mathrm{l})\end{array}$ & $\begin{array}{c}\text { SMMC7721 } \\
(\mathrm{mg} / \mathrm{l})\end{array}$ & $\begin{array}{c}\text { SMMC7721/ } \\
\text { ADM }(\mathrm{mg} / \mathrm{l})\end{array}$ & $\begin{array}{c}\text { SMMC7721/ADM+ } \\
\text { NSC348884 (mg/l) }\end{array}$ \\
\hline ADM & $0.24 \pm 0.07$ & $14.45 \pm 1.41^{\mathrm{aa}}$ & $1.52 \pm 0.28^{\mathrm{b}}$ & $1.58 \pm 0.22$ & $21.04 \pm 1.67^{\mathrm{c}}$ & $8.65 \pm 0.62^{\mathrm{d}}$ \\
DDP & $1.31 \pm 0.18$ & $5.17 \pm 0.29^{\mathrm{a}}$ & $2.83 \pm 0.19^{\mathrm{b}}$ & $3.5 \pm 0.17$ & $8.59 \pm 0.33^{\mathrm{cc}}$ & $5.12 \pm 0.31^{\mathrm{d}}$ \\
$5-\mathrm{Fu}$ & $8.54 \pm 0.16$ & $34.46 \pm 1.39^{\mathrm{a}}$ & $11.69 \pm 0.81^{\mathrm{bb}}$ & $6.66 \pm 0.26$ & $15.97 \pm 1.03^{\mathrm{c}}$ & $9.84 \pm 0.12^{\mathrm{dd}}$ \\
VCR & $0.48 \pm 0.03$ & $16.49 \pm 1.02^{\mathrm{aa}}$ & $7.82 \pm 0.11^{\mathrm{b}}$ & $0.32 \pm 0.02$ & $12.51 \pm 0.6^{\mathrm{cc}}$ & $6.74 \pm 0.1^{\mathrm{dd}}$ \\
VP-16 & $2.53 \pm 0.14$ & $26.38 \pm 0.96^{\mathrm{aa}}$ & $17.07 \pm 1.24^{\mathrm{bb}}$ & $3.86 \pm 0.25$ & $28.86 \pm 1.76^{\mathrm{c}}$ & $19.1 \pm 1.64^{\mathrm{d}}$ \\
\hline
\end{tabular}

Data are presented as the mean $\pm \mathrm{SD}(\mathrm{n}=6)$. ${ }^{\mathrm{a}} \mathrm{P}<0.05$ and ${ }^{\text {aa }} \mathrm{P}<0.01$ vs. HepG2; ${ }^{\mathrm{b}} \mathrm{P}<0.05$ and ${ }^{\mathrm{b} b} \mathrm{P}<0.01$ vs. HepG2/ADM; ${ }^{\mathrm{c}} \mathrm{P}<0.05$ and ${ }^{\mathrm{c}} \mathrm{P}<0.01$ vs. SMMC7721; ${ }^{\mathrm{d}} \mathrm{P}<0.05$ and ${ }^{\mathrm{dd}} \mathrm{P}<0.01$ vs. SMMC7721/ADM. $\mathrm{IC}_{50}$, half maximal inhibitory concentration; ADM, Adriamycin; DDP, diamminedichloroplatinum; 5-Fu, fluorouracil; VCR, vincristine sulfate; VP-16, etoposide; NSC348884, nucleophosmin inhibitor.

Reverse-transcription-quantitative polymerase chain reaction (RT-qPCR) analysis. RT-qPCR analysis was performed as described previously (33). Cells were plated in a 6-well plate at a concentration of $5 \times 10^{6}$ in $2 \mathrm{ml}$ of growth medium. Total RNA was extracted using TRIzol ${ }^{\circledR}$ (Takara Bio, Inc., Otsu, Japan) according to the manufacturer's protocol. Two micrograms of total RNA was reverse-transcribed into first-strand cDNA using Mx3005P. The following primers were used: NPM forward, 5'-GCAGTCGACGACACCAACATGGAA GATTCGATGGAC-3' and reverse, 5'-CGCGTTAACAAG AGACTTCCTCCACTG-3'; MDR1 forward, 5'-GGGGTA CCCCAGTCTCTACG-3' and reverse, 5'-CAAGCTTGT CCGACCTGAAGAG-3'; $\beta$-actin forward, 5'-TAAAGGGCA TCCTGGGCTACACT-3' and reverse, 5'-TTACTCCTTGGA GGCCATGTAGG-3'. PCR was performed for 35 cycles, each cycle was comprised of a denaturation step at $94^{\circ} \mathrm{C}$ for $45 \mathrm{sec}$, annealing at $50^{\circ} \mathrm{C}$ for $45 \mathrm{sec}$ and extension at $72^{\circ} \mathrm{C}$ for $45 \mathrm{sec}$, prior to a final extension step at $72^{\circ} \mathrm{C}$ for $10 \mathrm{~min}$. As a control, the housekeeping gene $\beta$-actin was amplified and quantified. Relative quantification of target gene expression was conducted using the $2^{-\triangle \Delta C q}$ method (34). RT-qPCR analysis was repeated $>3$ times.

Statistical analysis. All of the data were processed using the statistical software SPSS version 17.0 (SPSS Inc., Chicago, IL, USA). Samples were analyzed in triplicate, and three independent experiments were performed. Data are expressed as the mean \pm standard deviation, and differences between two groups were analyzed with the Student's $t$-test. $\mathrm{P}<0.05$ was considered to indicate a statistically significant difference.

\section{Results}

Determination of MDR in Hep G2/ADM and SMMC7721/ADM cells. ADM is a chemotherapeutic agent that is used for the primary treatment of tumors, including HCC (35). In the present study, ADM was applied to two HCC cell lines to generate MDR HepG2/ADM and SMMC7721/ADM cells. MDR HCC cell lines were generated over the course of 10 months. The $\mathrm{IC}_{50}$ values of different anticancer drugs in HepG2/ADM and SMMC7721/ADM cells were significantly higher when compared with that of their parental cells (Table I), and the CCK-8 assay revealed that HepG2/ADM and SMMC7721/ADM were not only resistant to ADM but also to multiple anticancer drugs, including DDP, 5-Fu, VCR and VP-16 (Table I). These results suggested that acquired MDR of HepG2/ADM and SMMC7721/ADM was successfully established.

NPM protein and $m R N A$ levels increased in Hep G2/ADM and SMMC7721/ADM cells when compared with their parental cells. As shown in Fig. 1A, NPM protein levels were significantly higher in the HepG2/ADM and SMMC7721/ADM cells when compared with their respective parental cells $(1.63 \pm 0.18$ vs. $0.99 \pm 0.25, \mathrm{P}<0.05 ; 2.39 \pm 0.19$ vs. $1.74 \pm 0.09$, $\mathrm{P}<0.05)$. RT-qPCR analysis demonstrated that NPM mRNA levels in the HepG2/ADM group were significantly higher when compared with that of the HepG2 group (1.64 \pm 0.23 vs. $1.01 \pm 0.2, \mathrm{P}<0.01)$, and the levels in the SMMC7721/ADM group were significantly higher than that of the SMMC7721 group $(2.51 \pm 0.08$ vs. $1.63 \pm 0.07, \mathrm{P}<0.01$; Fig. $1 \mathrm{~B})$. The results suggested that expression of NPM was upregulated in HepG2/ADM and SMMC7721/ADM cells when compared to their respective parental cells, and that these alterations occurred at the transcriptional level.

MDR-1 protein and $m R N A$ levels increased in HepG2/ADM and SMMC7721/ADM cells when compared to their parental cells. Western blotting and RT-qPCR analyses were used to determine the level of MDR expression in the two cell lines. MDR-1 protein and mRNA levels were significantly increased in the HepG2/ADM and SMMC7721/ADM cells when compared to their respective parental cells (MDR-1 protein, HepG2/ADM vs. HepG2, P<0.05; MDR-1 protein, SMMC7721/ADM vs. SMMC7721, P<0.01; MDR-1 mRNA, HepG2/ADM vs. HepG2, P<0.01; MDR-1 mRNA, SMMC7721/ADM vs. SMMC7721, P<0.01; Fig. 2). MDR-1 protein expression in each lane was normalized to $\beta$-actin expression.

Cell cycle phase distribution was significantly altered in HepG2/ADM and SMMC7721/ADM cells when compared to their parental cells. Cell cycle distribution was determined by flow cytometry analysis to examine differences between MDR HepG2/ADM and SMMC7721/ADM cells and their respective parental cells. The percentage of HepG2/ADM cells in the 

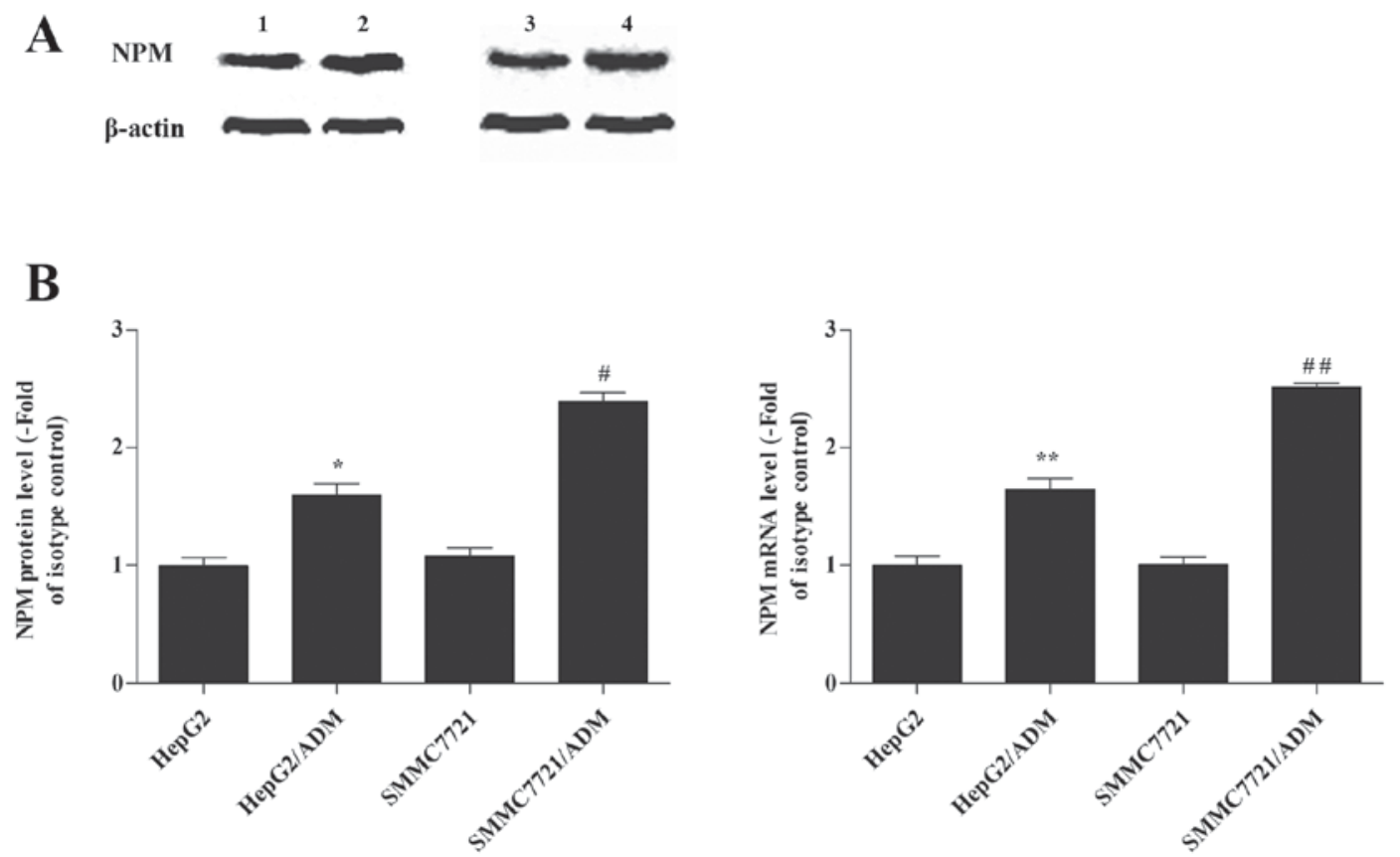

Figure 1. NPM protein and mRNA levels were increased in HepG2/ADM and SMMC7721/ADM cells when compared to their respective parental cells. (A) Western blotting images of NPM protein and $\beta$-actin expression. Lane 1, HepG2; lane 2, HepG2/ADM; lane 3, SMMC7721; lane 4, SMMC7721/ADM (B) Quantitative analysis of NPM protein and mRNA levels among all groups. $\beta$-actin was used as a loading control. ${ }^{*} \mathrm{P}<0.05$ and ${ }^{* *} \mathrm{P}<0.01 \mathrm{vs}$. HepG2; ${ }^{*} \mathrm{P}<0.05$ and ${ }^{\# \#} \mathrm{P}<0.01$ vs. SMMC7721. NPM, nucleophosmin; ADM, adriamycin.

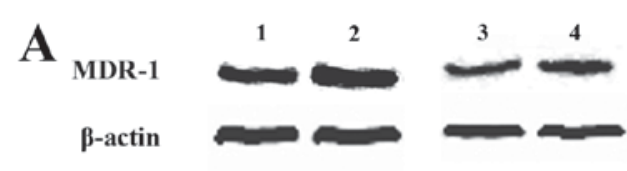

B
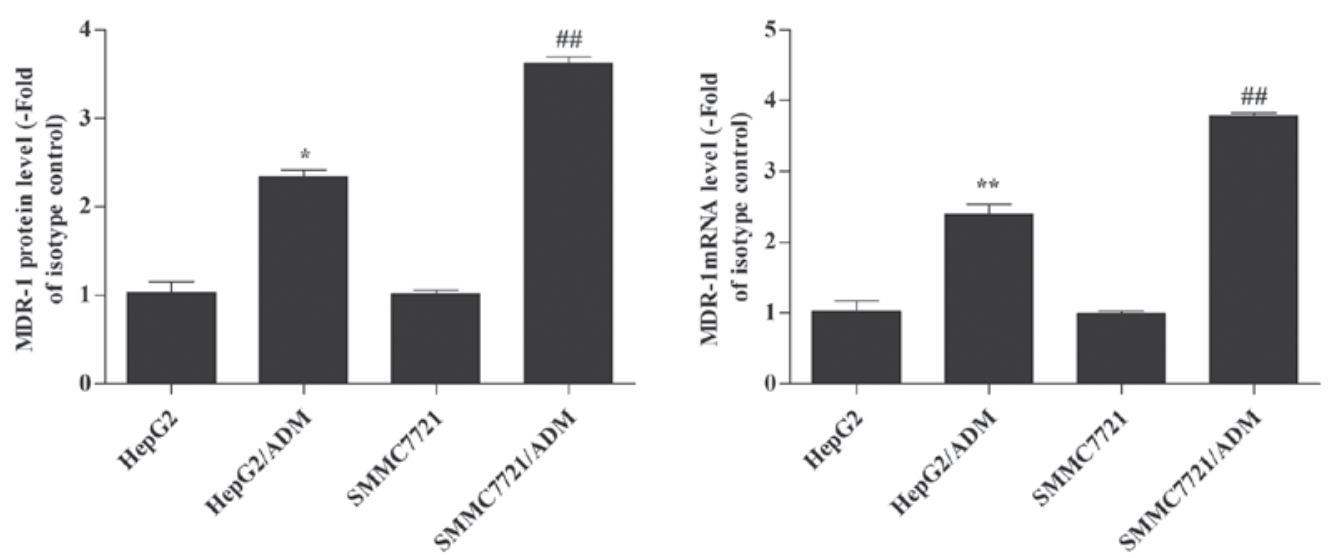

Figure 2. MDR-1 protein and mRNA levels were increased in HepG2/ADM and SMMC7721/ADM cells when compared to their respective parental cells. (A) Western blotting image for MDR-1 protein expression. $\beta$-actin was used as a loading control. Lane 1, HepG2; lane 2, HepG2/ADM; lane 3, SMMC7721; lane 4, SMMC7721/ADM. (B) Quantitative analysis of the expression of MDR-1 protein and mRNA among all groups. ${ }^{*} \mathrm{P}<0.05$ and ${ }^{* *} \mathrm{P}<0.01$ vs. HepG2. \#" $\mathrm{P}<0.01$ vs. SMMC7721. MDR-1, multidrug resistance gene 1; ADM, adriamycin.

$\mathrm{G}_{2} / \mathrm{M}$-phase and SMMC7721/ADM cells in the S-phase was significantly increased $\left(\mathrm{G}_{2} / \mathrm{M}\right.$-phase, HepG2/ADM vs. HepG2, $\mathrm{P}<0.01 ; \mathrm{G}_{2} / \mathrm{M}$-phase, SMMC7721/ADM vs. SMMC7721, $\mathrm{P}<0.05$; S-phase, HepG2/ADM vs. HepG2, P<0.01; S-phase, SMMC7721/ADM vs. SMMC7721, P<0.05; Table II), when compared with the parental cells. In addition, the percentage of HepG2/ADM and SMMC7721/ADM cells were significantly decreased at the $\mathrm{G}_{0} / \mathrm{G}_{1}$ phase (HepG2/ADM vs. HepG2,
$\mathrm{P}<0.05$; SMMC7721/ADM vs. SMMC7721, $\mathrm{P}<0.01$; Table II) when compared with the parental cells.

NSC348884 downregulates NPM levels. It has been previously reported that NSC348884 is a specific inhibitor of NPM (36). NSC348884 was used in the present study to determine whether downregulation of NPM reverses the MDR of HCC cell lines. MDR HCC cells were exposed to a variety of concentrations (1, 
Table II. Cell cycle distribution of parental and multidrug-resistant hepatocellular carcinoma cells.

\begin{tabular}{llcc}
\hline Cells & $\mathrm{G}_{0} / \mathrm{G}_{1}$ & $\mathrm{~S}$ & $\mathrm{G}_{2} / \mathrm{M}^{-}$ \\
\hline HepG2 & $66.69 \pm 2.26$ & $18.27 \pm 0.53$ & $14.98 \pm 0.73$ \\
HepG2/ADM & $59.97 \pm 1.37^{\mathrm{a}}$ & $12.67 \pm 0.29^{\mathrm{aa}}$ & $27.32 \pm 1.14^{\mathrm{aa}}$ \\
HepG2/ADM+NSC348884 & $63.48 \pm 1.83^{\mathrm{cc}}$ & $16.38 \pm 0.79^{\mathrm{c}}$ & $20.12 \pm 1.59^{\mathrm{c}}$ \\
SMMC7721 & $72.25 \pm 1.41$ & $17.48 \pm 0.39$ & $6.2 \pm 0.64$ \\
SMMC7721/ADM & $62.88 \pm 1.32^{\mathrm{bb}}$ & $33.32 \pm 1.41^{\mathrm{b}}$ & $3.61 \pm 0.65^{\mathrm{b}}$ \\
SMMC7721/ADM+NSC348884 & $68.21 \pm 1.04^{\mathrm{dd}}$ & $26.34 \pm 1.06^{\mathrm{dd}}$ & $5.43 \pm 0.34^{\mathrm{d}}$ \\
\hline
\end{tabular}

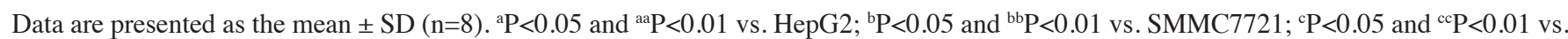
HepG2/ADM; ${ }^{\mathrm{d}} \mathrm{P}<0.05$ and ${ }^{\mathrm{dd}} \mathrm{P}<0.01$ vs. SMMC7721/ADM. ADM, Adriamycin; NSC348884, nucleophosmin inhibitor.

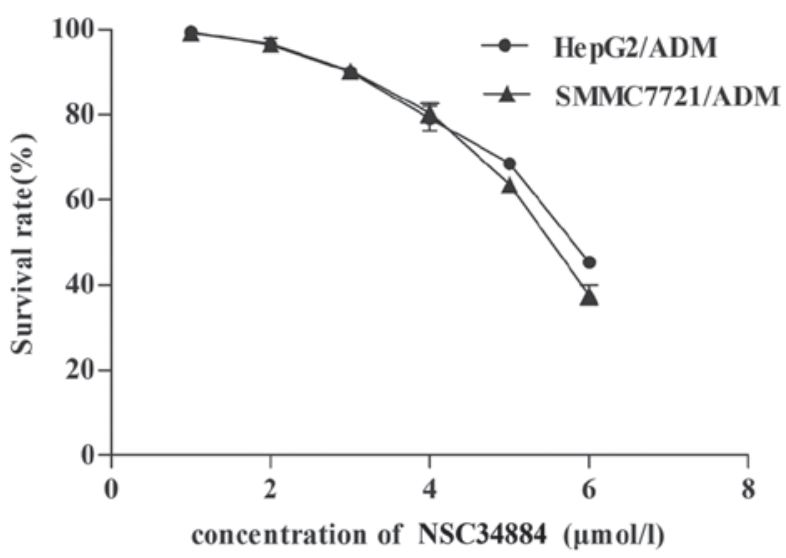

Figure 3. NSC348884 reduces the survival rate of multidrug resistant hepatocellular carcinoma cells. When $\leq 3 \mu \mathrm{mol} / 1$ NSC348884 was applied to HepG2/ADM and SMMC7721/ADM cells, no significant alterations in the rates of cell survival were observed. When $>3 \mu \mathrm{mol} / 1 \mathrm{NSC} 348884$ was applied, the cell survival rates markedly decreased. Data are presented as the mean \pm standard deviation of three independent experiments. ADM, adriamycin.

$2,3,4,5$ or $6 \mu \mathrm{mol} / \mathrm{l})$ of NSC348884. When cultured with $\leq 3 \mu \mathrm{mol} / 1 \mathrm{NSC} 348884$, HepG2/ADM and SMMC7721/ADM cells did not exhibit significant toxicity (Fig. 3). However, when cultured with $>3 \mu \mathrm{mol} / 1 \mathrm{NSC} 348884$, the cell survival rate of HepG2/ADM and SMMC7721/ADM cells markedly decreased (Fig. 3). As shown in Fig. 4, pretreatment of HepG2/ADM and SMMC7721/ADM cells with NSC348884, significantly decreased NPM protein and mRNA expression when compared to that of the parental cells (NPM protein, HepG2/ADM+NSC348884 vs. HepG2/ADM, P<0.05; NPM protein, SMMC7721/ADM+NSC348884 vs. SMMC7721/ADM, $\mathrm{P}<0.01$; N P M m R A , H e p 2 / A D M +NSC348884 vs. HepG2/ADM, P<0.01; NPM mRNA, SMMC7721/ADM+NSC348884 vs. SMMC7721/ADM, $\mathrm{P}<0.01$; Fig. 4).

NSC348884 reversed MDR in HepG2/ADM and SMMC7721/ADMcells. As demonstrated in Table I,HepG2/ADM and SMMC7721/ADM were resistant to ADM, as well as DDP, 5 -Fu, VCR and VP16 anticancer drugs. The $\mathrm{IC}_{50}$ values were $5.17 \pm 0.29$ and $34.46 \pm 1.39 \mathrm{mg} / \mathrm{l}$ in HepG2/ADM cells treated with
DDP and 5-Fu, respectively, and 8.59 \pm 0.33 and $15.97 \pm 1.03 \mathrm{mg} / \mathrm{l}$ in SMMC7721/ADM treated with DDP and 5-Fu, respectively (Table I). HepG2 and SMMC7721 cells were more sensitive to these drugs, with $\mathrm{IC}_{50}$ values of $1.31 \pm 0.18$ and $8.54 \pm 0.16 \mathrm{mg} / \mathrm{l}$ in HepG2 cells treated with DDP and 5-Fu, respectively, and $3.5 \pm 0.17$ and $6.66 \pm 0.26 \mathrm{mg} / \mathrm{l}$ in SMMC7721 cells treated with DDP and 5-Fu, respectively. Pretreatment of HepG2/ADM and SMMC7721/ADM cells with $3 \mu \mathrm{mol} / 1 \mathrm{NSC} 348884$ was associated with increased sensitivity to these agents. The $\mathrm{IC}_{50}$ values were $2.83 \pm 0.19$ and $11.69 \pm 0.81 \mathrm{mg} / \mathrm{l}$ in HepG2/ADM+NSC348884 cells treated with DDP and 5-Fu, respectively, and 5.12 \pm 0.31 and $9.84 \pm 0.12 \mathrm{mg} / \mathrm{l}$ in SMMC7721/ADM+NSC348884 cells treated with DDP and 5-Fu, respectively (Table I). In addition, pretreatment of HepG2/ADM and SMMC7721/ADM cells with $3 \mu \mathrm{mol} / 1$ NSC348884, was associated with a significant decrease in MDR-1 protein and mRNA levels in the HepG2/ADM+NSC348884 and SMMC7721/ADM+NSC348884 cells when compared with the HepG2/ADM and SMMC7721/ADM cells (MDR-1 protein, HepG2/ADM+NSC348884 vs. HepG2/ADM, $\mathrm{P}<0.05$; MDR-1 protein, SMMC7721/ADM+NSC348884 vs. SMMC7721/ADM, P<0.01; MDR-1 mRNA, HepG2/ADM +NSC348884 vs. HepG2/ADM, P<0.01; MDR-1 mRNA, SMMC7721/ADM+NSC348884 vs. SMMC7721/ADM, P<0.01; Fig. 5). The quantity of product in each lane was normalized to $\beta$-actin expression. Alterations in the cell cycle distribution of HepG2/ADM and SMMC7721/ADM cells were significantly reversed following treatment with NSC348884 (Table II). The percentage of HepG2/ADM cells in $\mathrm{G}_{2}$ /M-phase and SMMC7721/ ADM cells in S-phase was significantly increased, when compared with the parental cells. In addition, the percentage of HepG2/ADM and SMMC7721/ADM cells were significantly decreased at the $\mathrm{G}_{0} / \mathrm{G}_{1}$ phase when compared with the parental cells. These results suggest that NSC348884 may reverse the MDR of HepG2/ADM and SMMC7721/ADM cells.

The effect of NPM on P-gp expression. In order to investigate the effect of NPM on P-gp expression, western blot analysis was performed (Fig. 6). It was revealed that P-gp expression was significantly higher in HepG2/ADM and SMMC7721/ADM cells when compared with the parental cells $(\mathrm{P}<0.01$ and $\mathrm{P}<0.01$, respectively; Fig. 6). By contrast, when HepG2/ADM and SMMC7721/ADM cells were pretreated with NSC348884, $\mathrm{P}-$ gp expression was significantly reduced $(\mathrm{P}<0.01$ and $\mathrm{P}<0.01$, respectively; Fig. 6). 

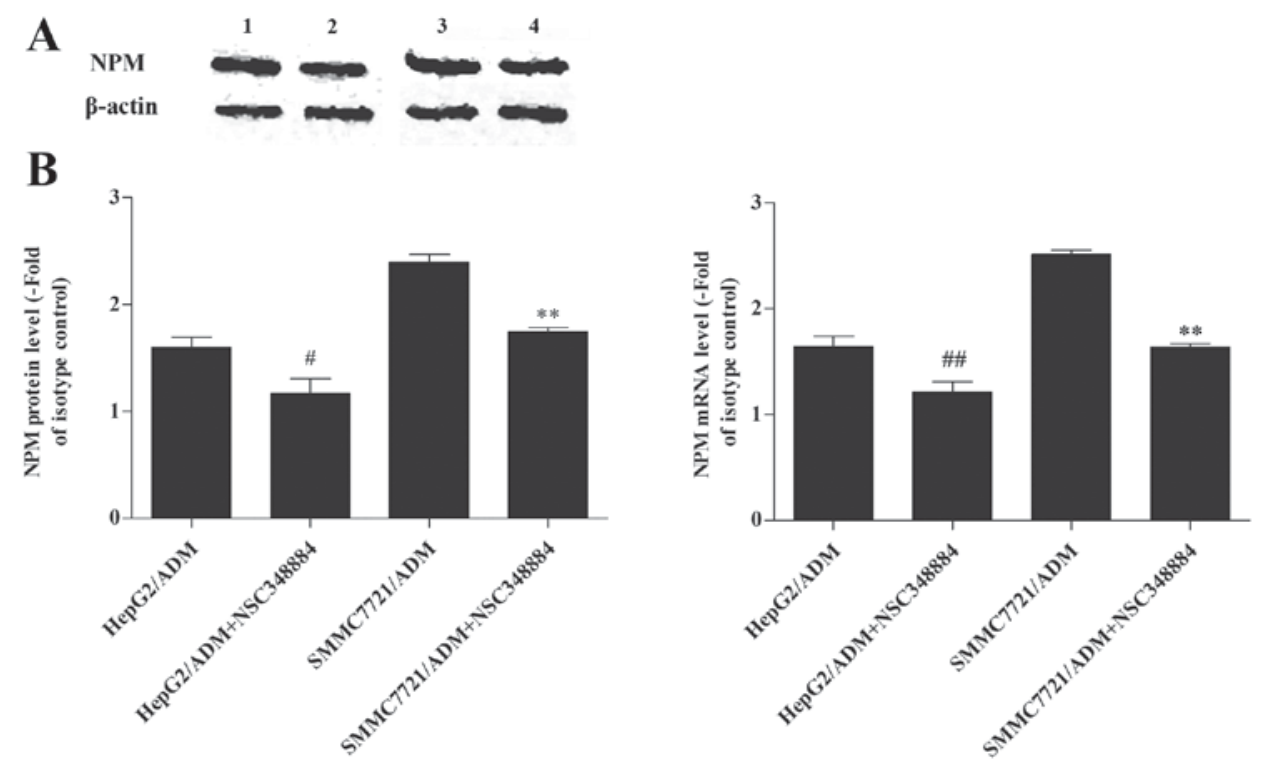

Figure 4. Pretreatment of HepG2/ADM and SMMC7721/ADM cells with NSC348884 decreased NPM expression when compared to the untreated cells. (A) Western blotting images of NPM and $\beta$-actin protein expression levels. Lane 1, HepG2/ADM; lane 2, HepG2/ADM+NSC348884; lane 3, SMMC7721/ ADM; lane 4, SMMC7721/ADM+NSC348884. (B) Quantitative analysis of NPM protein and mRNA expression among all groups. $\beta$-actin was used as a loading control. ${ }^{\#} \mathrm{P}<0.05$ and ${ }^{\# \#} \mathrm{P}<0.01$ vs. HepG2/ADM; ${ }^{* *} \mathrm{P}<0.01$ vs. SMMC7721/ADM. ADM, adriamycin; NPM, nucleophosmin.

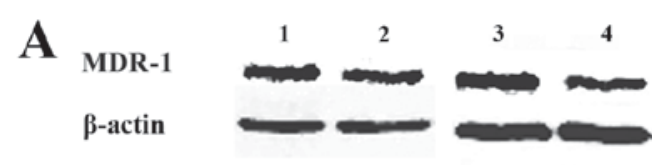

B
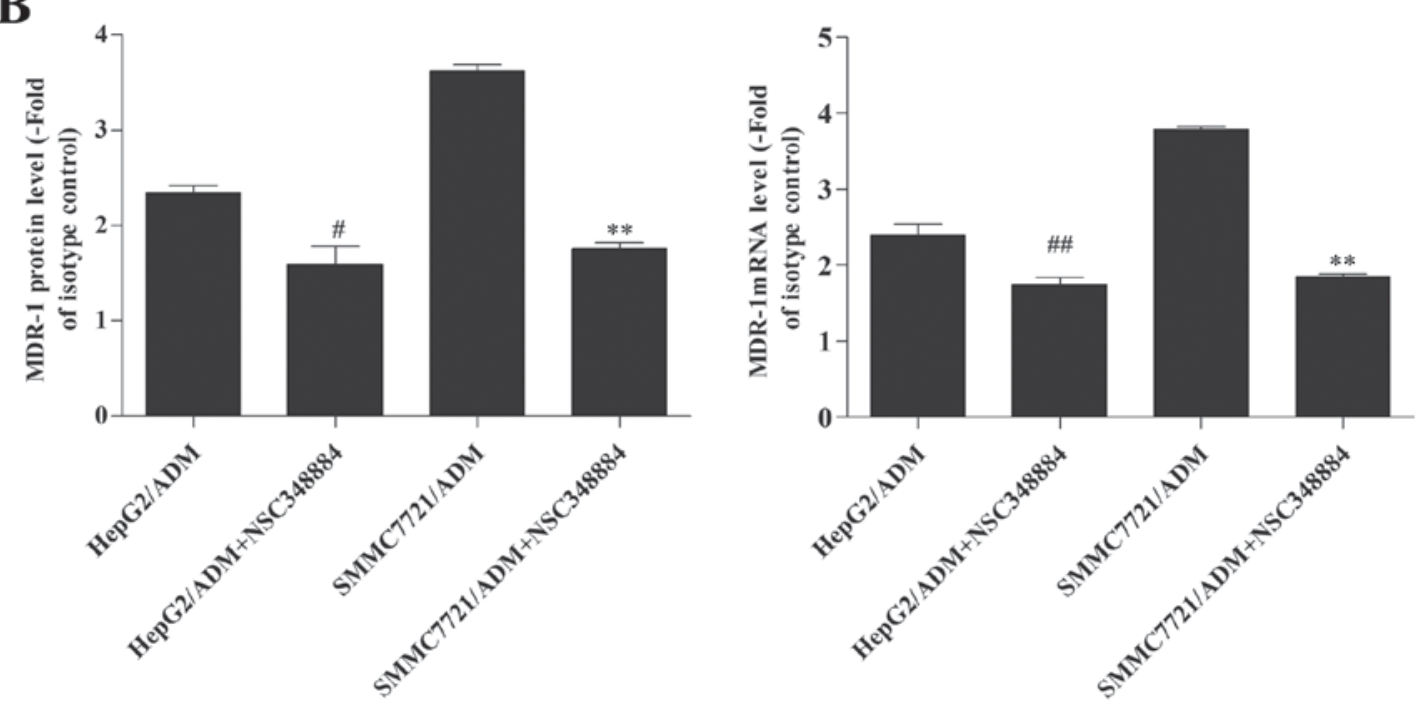

Figure 5. Pretreatment of HepG2/ADM and SMMC7721/ADM cells with NSC348884 decreased MDR-1 expression when compared to the parental cells. (A) Western blotting images of MDR-1 protein expression. $\beta$-actin was used as a loading control. Lane 1, HepG2/ADM; lane 2, HepG2/ADM+NSC348884; lane 3, SMMC7721/ADM; lane 4, SMMC7721/ADM+NSC348884. (B) Quantitative analysis of the expression of MDR-1 protein and mRNA among all groups. ${ }^{\#} \mathrm{P}<0.05$ and ${ }^{\# \#} \mathrm{P}<0.01$ vs. HepG2/ADM; * $\mathrm{P}<0.01$ vs. SMMC7721/ADM. ADM, Adriamycin; MDR-1, multidrug resistance gene 1.

\section{Discussion}

MDR is characterized by the development of anticancer drug resistance, which may lead to the development of resistance to other pharmacokinetic and structurally unrelated drugs $(37,38)$. For a number of years, MDR has been a major issue for scientists and clinicians in the treatment of cancer, however an effective solution has remained elusive.
Due to the difficulties encountered in the reversal of MDR, alternative methods to overcome MDR in cancer cells are continuously being investigated. Recently, NPM has received significant interest due to its association with ADM-resistant cells (39). NPM, also known as B23, NO38 or Numatrin, is a $38-\mathrm{kDa}$ estrogen-regulated nucleolar phosphoprotein that shuttles between the nucleus and cytoplasm (40). NPM function has been implicated in a number of cellular processes, 


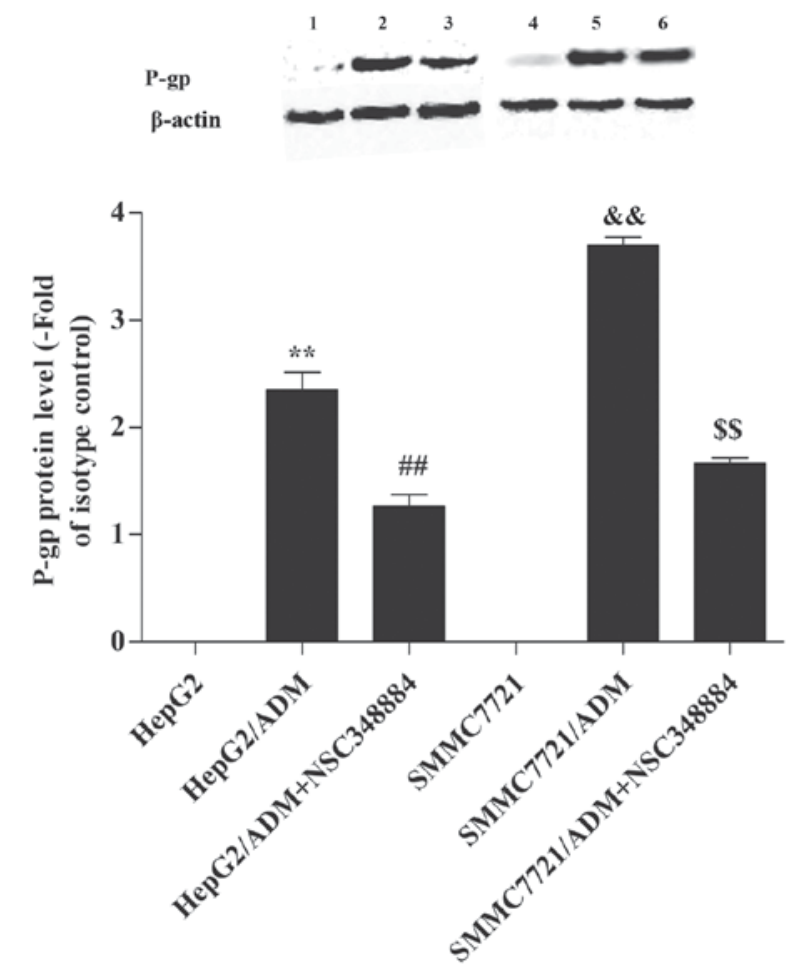

Figure 6. Effect of nucleophosmin on P-gp expression. A high level of P-gp expression was detected in HepG2/ADM and SMMC7721/ADM cells. However, when HepG2/ADM and SMMC7721/ADM cells were pretreated with NSC348884, the P-gp level was significantly decreased. Lane 1, HepG2; lane 2,HepG2/ADM; lane 3, HepG2/ADM+NSC348884; lane 4, SMMC7721 lane 5, SMMC7721/ADM; lane 6, SMMC7721/ADM+NSC348884. ${ }^{* *} \mathrm{P}<0.01$ vs. HepG2; ${ }^{\#} \mathrm{P}<0.01$ vs. HepG2/ADM; ${ }^{\&} \mathrm{P}<0.01$ vs. SMMC7721 and ${ }^{\$} \mathrm{P}<0.01$ vs. SMMC7721/ADM. ADM, Adriamycin; P-gp, P-glycoprotein.

including ribosome shuttling between precursor proteins in the cytoplasm and nucleus, nuclear protein chaperone activity, the maintenance of genomic stability and the indirect regulation of growth and proliferation $(39,41,42)$. NPM overexpression has frequently been associated with tumor progression, and may be a marker for some cancers, including gastric, ovarian, prostate (42) and Ewing's sarcoma (43). A previous study reported that NPM was highly expressed in human MDR gastric cancer cell lines (44), radiotherapy-resistant HeLa cells $(45,46)$ and in the MCF-7 breast cancer cell line where upregulated NPM expression enhances interferon regulating factor 1 mediated estrogen-resistance (47). However, the effect of NPM downregulation on the reversal of MDR in HCC, as well as the underlying molecular mechanisms involved, remain unknown. Therefore, the present study was designed to investigate the effect of NPM downregulation on MDR, and the molecular mechanisms involved in this process. The results demonstrated that NPM expression was significantly increased in MDR HCC cell lines when compared to that of their parental cells.

To identify the mechanisms involved in the development of MDR in HepG2/ADM and SMMC7721/ADM cells, the cell cycle distribution was analyzed by flow cytometry. The percentage of MDR HepG2/ADM and SMMC7721/ ADM cells was markedly decreased in the $G_{0} / G_{1}$ phase and increased in the $S$ and $G_{2} / M$ phases when compared to their parental cells. This may have been responsible for the reduced cell proliferation ability (date not shown). In addition, delayed cell cycle progression may facilitate the removal of specific cytotoxic agents from the cell, thus leading to MDR in the cells.

An improved understanding of the possible molecular mechanisms and signaling pathways involved in MDR is important to overcome MDR and improve chemotherapeutic efficacy in patients with HCC. Multiple hypotheses have been proposed regarding the mechanisms underlying the development of MDR, including the involvement of P-gp, which is encoded by the MDR1 gene (26). Previous studies have demonstrated that $\mathrm{P}-\mathrm{gp}$ relies on the actin cytoskeleton for its localization in lipid rafts on the cell membrane, thereby influencing drug influx and possibly counteracting uptake $(48,49)$. The action of P-gp as a drug efflux pump for therapies including ADM, docetaxel, paclitaxel and daunorubicin (50), has led to the development of chemosensitizing agents including cyclosporine, verapamil and quinine, which competitively or noncompetitively inhibit this protein (51). The expression of $\mathrm{P}-\mathrm{gp}$ is increased in drug-resistant tumors of the colon, kidney and adrenal gland, as well as in some tumors that have acquired MDR following chemotherapy (52). Excessive P-gp has been demonstrated to bind and transport anticancer drugs through ATP-dependent anticancer drug efflux pumps, leading to an increased efflux of the anticancer agent from the cancer cells, and a lower intracellular concentration $(26,31,53)$. The results of the present study demonstrated that P-gp expression was increased in MDR HepG2/ADM and SMMC7721/ADM cells when compared with their parental cells, indicating that MDR of HepG2/ADM and SMMC7721/ADM cells may be attributed to the overexpression of P-gp.

In order to further explore the role of NPM in the HCC MDR cell lines, NSC348884, a specific inhibitor of NPM, was applied to investigate whether downregulation of NPM may reverse MDR in HCC cell lines. Previous studies have demonstrated that application of NSC348884 suppressed the proliferation of prostate, colon, breast, lung and lymphoma tumor cells, thereby enhancing ADM sensitivity (36). Following NSC348884 treatment of MDR HCC cells in the present study, cellular resistance to anticancer drugs was reversed, and corresponding alterations in the cell cycle distributions were observed. Further experiments suggested that NSC348884 may reverse MDR, via inhibition of P-gp function.

NSC348884 significantly reversed HCC MDR in the present study. The results implied that NSC348884 may be effective in reversing MDR in vitro. In addition, RT-qPCR and western blot analysis revealed that the expression of P-gp at the mRNA and protein level were decreased. Reduced expression of P-gp at the transcriptional and translational levels has been proposed to be one of the mechanisms for certain modulators or agents to reverse the MDR phenotype (54).

In conclusion, the results of the present study have provided evidence demonstrating that NPM protein and mRNA levels were increased in HepG2/ADM and SMMC7721/ADM cells when compared to that of their parental cells. In addition, treatment of cells with a specific inhibitor of NPM (NSC348884) was able to reverse the MDR of HepG2/ADM and SMMC7721/ ADM cells, potentially via the downregulation of P-gp expression. The results suggest that NPM may be involved in MDR 
of HCC. It is a novel MDR reversal agent and may be a potential adjuvant agent for tumor chemotherapy. However, further research is required to optimize NPM exposure, and to determine the mechanisms underlying how downregulation of NPM leads to enhanced sensitivity of MDR HCC cells to anticancer drugs.

\section{Acknowledgements}

The present study was supported by grants from the Nature Scientific Foundation of Shanxi Province (grant no. 2011021035-3) and the Scientific Foundation of Shanxi P rovincial Health Department (grant no. 200810).

\section{References}

1. Schlageter M, Terracciano LM, D'Angelo S and Sorrentino P Histopathology of hepatocellular carcinoma. World J Gastroenterol 20: 15955-15964, 2014.

2. Gu W, Fang FF, Li B, Cheng BB and Ling CQ: Characterization and resistance mechanisms of A 5-fluorouracil resistance hepatocellular carcinoma cell line. Asian Pac J Cancer Prev 13: 4807-4814, 2012.

3. Verheggen C, Almouzni G and Hernandez-Verdun D: The ribosomal RNA processing machinery is recruited to the nucleolar domain before RNA polymerase I during Xenopus laevis development. J Cell Biol 149: 293-306, 2000.

4. Huang N, Negi S, Szebeni A and Olson MO: Protein NPM3 interacts with the multifunctional nucleolar protein $\mathrm{B} 23 /$ nucleophosmin and inhibits ribosome biogenesis. J Biol Chem 280: 5496-5502, 2005.

5. Okuda M, Horn HF, Tarapore P, Tokuyama Y, Smulian AG, Chan PK, Knudsen ES, Hofmann IA, Snyder JD, Bove KE and Fukasawa K: Nucleophosmin/B23 is a target of CDK2/cyclin E in centrosome duplication. Cell 103: 127-140, 2000.

6. Okuda M: The role of nucleophosmin in centrosome duplication. Oncogene 21: 6170-6174, 2002.

7. Grisendi S, Bernardi R, Rossi M, Cheng K, Khandker L, Manova K and Pandolfi PP: Role of nucleophosmin in embryonic development and tumorigenesis. Nature 437: 147-153, 2005.

8. Hingorani K, Szebeni A and Olson MO: Mapping the functional domains of nucleolar protein B23. J Biol Chem 275: 24451-24457, 2000.

9. Szebeni A, Hingorani K, Negi S and Olson MO: Role of protein kinase CK2 phosphorylation in the molecular chaperone activity of nucleolar protein b23. J Biol Chem 278: 9107-9115, 2003.

10. Colombo E, Marine JC, Danovi D, Falini B and Pelicci PG Nucleophosmin regulates the stability and transcriptional activity of p53. Nat Cell Biol 4: 529-533, 2002.

11. Li J,Zhang X, Sejas DP, Bagby GC and Pang Q: Hypoxia-induced nucleophosmin protects cell death through inhibition of $\mathrm{p} 53$ J Biol Chem 279: 41275-41279, 2004

12. Maiguel DA, Jones L, Chakravarty D, Yang C and Carrier F: Nucleophosmin sets a threshold for 553 response to UV radiation. Mol Cell Biol 24: 3703-3711, 2004.

13. Itahana K, Bhat KP, Jin A, Itahana Y, Hawke D, Kobayashi R and Zhang Y: Tumor suppressor ARF degrades B23, a nucleolar protein involved in ribosome biogenesis and cell proliferation. Mol Cell 12: 1151-1164, 2003.

14. Bertwistle D, Sugimoto M and Sherr CJ: Physical and functional interactions of the Arf tumor suppressor protein with nucleophosmin/B23. Mol Cell Biol 24: 985-996, 2004.

15. Brady SN, Yu Y, Maggi LB Jr and Weber JD: ARF impedes NPM/B23 shuttling in an Mdm2-sensitive tumor suppressor pathway. Mol Cell Biol 24: 9327-9338, 2004.

16. Feuerstein $\mathrm{N}$ and Mond JJ: 'Numatrin,' a nuclear matrix protein associated with induction of proliferation in B lymphocytes. J Biol Chem 262: 11389-11397, 1987.

17. Feuerstein N, Spiegel S and Mond JJ: The nuclear matrix protein, numatrin (B23), is associated with growth factor-induced mitogenesis in Swiss 3T3 fibroblasts and with T lymphocyte proliferation stimulated by lectins and anti-T cell antigen receptor antibody. J Cell Biol 107: 1629-1642, 1988.

18. Pulford K, Morris SW and Mason DY: Anaplastic lymphoma kinase proteins and malignancy. Curr Opin Hematol 8: 231-236, 2001.
19. Tanaka M, Sasaki H, Kino I, Sugimura T and Terada M: Genes preferentially expressed in embryo stomach are predominantly expressed in gastric cancer. Cancer Res 52: 3372-3377, 1992.

20. Nozawa Y, Van Belzen N, Van der Made AC, Dinjens WN and Bosman FT: Expression of nucleophosmin/B23 in normal and neoplastic colorectal mucosa. J Pathol 178: 48-52, 1996.

21. Tsui KH, Cheng AJ, Chang Pe, Pan TL and Yung BY: Association of nucleophosmin/B23 mRNA expression with clinical outcome in patients with bladder carcinoma. Urology 64: 839-844, 2004.

22. Subong EN, Shue MJ, Epstein JI, Briggman JV, Chan PK and Partin AW: Monoclonal antibody to prostate cancer nuclear matrix protein (PRO:4-216) recognizes nucleophosmin/B23. Prostate 39: 298-304, 1999.

23. Onda M, Emi M, Yoshida A, Miyamoto S, Akaishi J, Asaka S, Mizutani K, Shimizu K, Naqahama M, Ito K, et al: Comprehensive gene expression profiling of anaplastic thyroid cancer with cDNA microarray of 25344 genes. Endocr Relat Cancer 11: 843-854, 2004.

24. Zhang Y: The ARF-B23 connection: implications for growth control and cancer treatment. Cell Cycle 3: 259-262, 2004.

25. Schnittger S, Schoch C, Kern W, Mecucci C, Tschulik C, Martelli MF, Haferlach T, Hiddemann W and Falini B: Nucleophosmin gene mutations are predictors of favorable prognosis in acute myelogenous leukemia with a normal karyotype. Blood 106: 3733-3739, 2005.

26. Gottesman MM, Fojo T and Bates SE: Multidrug resistance in cancer: Role of ATP-dependent transports. Nat Rev Cancer 2: 48-58, 2002.

27. Ambudkar SV, Kimchi-Sarfaty C, Sauna ZE and Gottesman MM: P-glycoprotein: From genomics to mechanism. Oncogene 22: 7468-7485, 2003

28. Glavinas H, Krajcsi P, Cserepes J and Sarkadi B: The role of $\mathrm{ABC}$ transporters in drug resistance, metabolism and toxicity. Curr Drug Deliv 1: 27-42, 2004.

29. Varma MV, Ashokraj Y,Dey CS and Panchagnula R:P-glycoprotein inhibitors and their screening: A perspective from bioavailability enhancement. Pharmacol Res 48: 347-359, 2003.

30. Johnson WW: P-glycoprotein-mediated efflux as a major factor in the variance of absorption and distribution of drugs: Modulation of chemotherapy resistance. Methods Find Exp Clin Pharmacol 24: 501-514, 2002.

31. Fojo $\mathrm{T}$ and Bates S: Strategies for reversing drug resistance. Oncogene 22: 7512-7523, 2003.

32. Nguyen T, Zhang XD and Hersey P: Relative resistance of fresh isolates of melanoma to tumor necrosis factor-related apoptosis-inducing ligand (TRAIL)-induced apoptosis. Clin Cancer Res 7: 966s-973s, 2001.

33. Li X, Zhao H, Wu Y, Zhang S, Zhao X, Zhang Y, Wang J, Wang J and Liu $\mathrm{H}$ : Up-regulation of hypoxia-inducible factor-1 $\alpha$ enhanced the cardioprotective effects of ischemic postconditioning in hyperlipidemic rats. Acta Biochim Biophys Sin (Shanghai) 46: 112-118, 2014.

34. Livak KJ and Schmittgen TD: Analysis of relative gene expression data using real-time quantitative PCR and the 2(-Delta Delta C(T)) Method. Methods 25: 402-408, 2001.

35. Minotti G, Menna P, Salvatorelli E, Cairo G and Gianni L: Anthracyclines: Molecular advances and pharmacologic developments in antitumor activity and cardiotoxicity. Pharmacol Rev 56: 185-229, 2004.

36. Qi W, Shakalya K, Stejskal A, Goldman A and Beeck S, Cooke L and Mahadevan D: NSC348884, a nucleophosmin inhibitor disrupts oligomer formation and induces apoptosis in human cancer cells. Oncogene 2: 4210-4220, 2008.

37. Pérez-Tomás R: Multidrug resistance: Retrospect and prospects in anti-cancer drug treatment. Curr Med Chem 13: 1859-1876, 2006.

38. Daniel C, Bell C, Burton C, Harguindey S, Reshkin SJ and Rauch C: The role of proton dynamics in the development and maintenance of multidrug resistance in cancer. Biochim Biophys Acta 1832: 606-617, 2013.

39. Yung BY: Oncogenic role of nucleophosmin/B23. Chang Gung Med J 30: 285-293, 2007.

40. Lam L, Aktary Z, Bishay M, Werkman C, Kuo CY, Heacock M, Srivastava N, Mackey JR and Pasdar M: Regulation of subcellular distribution and oncogenic potential of nucleophosmin by plakoglobin. Oncogenesis 1: e4, 2012.

41. Skaar TC, Prasad SC, Sharareh S, Lippman ME, Brunner N and Clarke R: Two-dimensional gel electrophoresis analyses identify nucleophosmin as an estrogen regulated protein associated with acquired estrogen-independence in human breast cancer cells. J Steroid Biochem Mol Biol 67: 391-402, 1998. 
42. Grisendi S, Mecucci C, Falini B and Pandolfi PP: Nucleophosmin and cancer. Nat Rav Cancer 6: 493-505, 2006.

43. Kikuta K, Tochigi N, Shimoda T, Yabe H, Morioka H, Toyama Y, Hosono A, Beppu Y, Kawai A, Hirohashi S and Kondo T: Nucleophosmin as a candidate prognostic biomarker of Ewing's sarcoma revealed by proteomics. Clin Cancer Res 15: 2885-2894, 2009.

44. Yang YX, Hu HD, Zhang DZ and Ren H: Identification of proteins responsible for the development of adriamycin resistance in human gastric cancer cells using comparative proteomics analysis. J Biochem Mol Biol 40: 853-860, 2007.

45. Wu MH, Chang JH, Chou CC and Yung BY: Involvement of nucleophosmin-B23 in the response of HeLa cells to UV irradiation. Int J Cancer 97: 297-305, 2002.

46. Wu MH, Chang JH and Yung BY: Resistance to UV-induced cell-killing in nucleophosmin/B23 over-expressed NIH3T3 fibroblasts: Enhancement of DNA repair and up-regulation of PCNA in association with nucleophosmin/B23 over-expression. Carcinogenesis 23: 93-100, 2002.

47. Clarke R, Liu MC, Bouker KB, Gu Z, Lee RY, Zhu Y, Skaar TC, Gomez B, O'Brien K, Wang Y, et al: Antiestrogen resistance in breast cancer and the role of estrogen receptor signaling. Oncogene 22: 7316-7339, 2003.
48. Zhang L, Xiao R, Xiong J, Leng J, Ehtisham A, Hu Y, Ding Q, $\mathrm{Xu} \mathrm{H}$, Liu S, Wang J, et al: Activated ERM Protein plays a critical role in drug resistance of MOLT4 cells induced by CCL25. PLoS One 8: e52384, 2013.

49. Meszaros P, Hummel I, Klappe K, Draghiciu O, Hoekstra D and Kok JW: The fuction of the ATP-bnding cassette (ABC) transporter ABCB1 is not susceptible to actin disruption. Biochim Biophys Acta 1828: 340-351, 2013

50. Avendaño $\mathrm{C}$ and Menéndez JC: Inhibitors of multidrug resistance to antitumor agents (MDR). Curr Med Chem 9: 159-193, 2002.

51. Leonard GD, Fojo T and Bates SE: The role of ABC transporters in clinical practice. Oncologist 8: 411-424, 2003.

52. Tsuruo T: Molecular cancer therapeutics: Recent progress and targets in drug resistance. Intern Med 42: 237-243, 2003.

53. Ozben T: Mechanism and strategies to overcome multiple drug resistance in cancer. FEBS Lett 580: 2903-2909, 2006

54. Hu YP, Pourquer P, Doignon F, Crouzet M and Robert J: Effects of modulators of multidrug resistance on the expression of the MDR1 gene in human KB cells in culture. Anticancer Drugs 7: 738-744, 1996. 